\title{
Concept of modular flexure-based mechanisms for ultra-high precision robot design
}

\author{
M. Richard and R. Clavel \\ Ecole Polytechnique Fédérale de Lausanne, Laboratoire de Systèmes Robotiques (LSRO), \\ Station 9, 1015 Lausanne, Switzerland
}

Received: 25 February 2011 - Revised: 20 April 2011 - Accepted: 2 May 2011 - Published: 10 May 2011

\begin{abstract}
This paper introduces a new concept of modular flexure-based mechanisms to design industrial ultra-high precision robots, which aims at significantly reducing both the complexity of their design and their development time. This modular concept can be considered as a robotic Lego, where a finite number of building bricks is used to quickly build a high-precision robot. The core of the concept is the transformation of a 3-D design problem into several 2-D ones, which are simpler and well-mastered. This paper will first briefly present the theoretical bases of this methodology and the requirements of both types of building bricks: the active and the passive bricks. The section dedicated to the design of the active bricks will detail the current research directions, mainly the maximisation of the strokes and the development of an actuation sub-brick. As for the passive bricks, some examples will be presented, and a discussion regarding the establishment of a mechanical solution catalogue will conclude the section. Last, this modular concept will be illustrated with a practical example, consisting in the design of a 5-degree of freedom ultra-high precision robot.
\end{abstract}

\section{Motivation}

The current trend in numerous industrial domains is to miniaturise products, mainly microelectronic, optic and biomedical devices; the design of industrial robots capable of performing micromanipulation and micro-assembly tasks with a sub-micrometric precision is thus becoming a crucial need (Tolfree, 2006). As the use of flexure joints is compulsory to meet these precision requirements, a key aspect of the robot development consists in the design of the flexure-based mechanisms. Although this step is now widely investigated for planar and low-DOF (Degrees of Freedom) compliant structures, the development of a whole flexure-based robot is still infrequent, especially in the industrial context. Two examples of industrial prototypes are the Delta ${ }^{3}$ robot family, which consists in an adaptation of the classical Delta kinematics for $\mu$-Wire Electro-Discharge Machining (3 DOF in translation, strokes: $\pm 2 \mathrm{~mm}$, resolution: $5 \mathrm{~nm}$, see Fig. 1) (Bacher et al., 2002; Bottinelli et al., 2002), and the 6-DOF Sigma 6 robot, intended to perform active optical alignment (strokes: $\pm 4 \mathrm{~mm}$ and $\pm 4^{\circ}$, resolution: $5 \mathrm{~nm}$ on the linear axes) (Helmer et al., 2004; Helmer, 2006). Nonetheless, the

Correspondence to: M. Richard (murielle.richard@epfl.ch) development time of such ultra-high precision machines, and thus their time-to-market, is considerably high. Furthermore, if the industrial specifications are modified, requiring for instance to add a degree of freedom or to change the position of a rotation centre, the whole design process has to be restarted from the beginning.

This paper thus presents a concept of modular flexurebased mechanisms, which allows to rapidly design an ultrahigh precision robot and to easily change its mobility. This approach can be compared to a robotic Lego, where a finite number of building bricks can be chosen and assembled within a small amout of time to create the desired machine (Richard and Clavel, 2010). Besides the kinematical aspects of the robot, which will not be exposed here, the concept makes use of mechanical bricks, which consist in planar flexure-based mechanisms. This modular concept thus allows to transform a 3-D mechanical design problem into several 2-D ones: the flexure-based structures resulting from the 3 -D conception are complex and scarcely flexible, whereas the 2-D solutions are simple, well-mastered and easy to machine monolithically. Likewise, both the kinematical and mechanical designs are considerably simplified thanks to the use of solution catalogues. The robots resulting from this methodology thus achieve similar high performances as more traditionally designed machines, with a significantly shortened development time. 


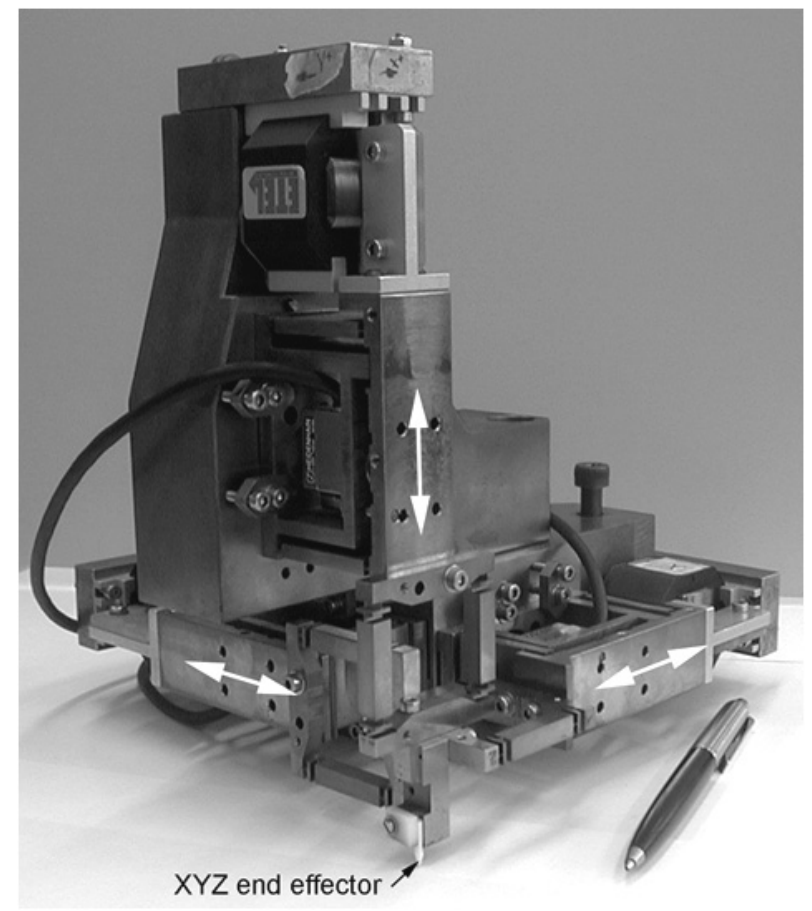

Figure 1. Delta ${ }^{3}$ II robot (3 translational DOF, strokes: $\pm 2 \mathrm{~mm}$, resolution: $5 \mathrm{~nm}$ ) (Bacher et al., 2002).

The current research on this modular concept will be presented in this paper: first, a brief description of the theoretical aspects will allow to establish the terminology and the chief specifications of the flexure-based building bricks. Then, the latest research directions will be presented, with a special focus on the maximisation of the flexure strokes and the development of an actuation sub-brick. Last, the application of this modular concept to design a 5-DOF ultra-high precision robot will illustrate the potentialities of this new approach.

\section{Concept of modular flexure-based robot}

A parallel robot resulting from this modular concept is composed of one to three kinematic chains and can be symbolised by a cube: the chains are disposed orthogonally along its faces, while the end-effector is located on one of its corners. The design of this robot makes use of a finite number of building bricks: these consist in 2-D flexure-based mechanisms and present two states: active and passive. As their name indicates, the role of the active bricks is to actuate from one to three degrees of freedom, making use of linear actuators only. The translations are obtained by the synchronous displacement of the actuators, whereas the rotations are performed by their differential motion. As for the passive bricks, their function is to link the output of the active bricks to the end-effector of the robot, thus performing a transmission of the actuated motion. Each kinematical chain of the modular

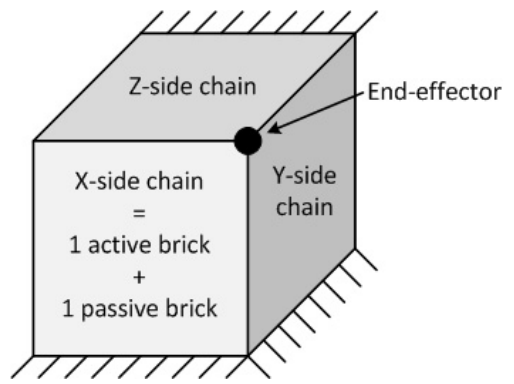

Figure 2. Symbolism of the parallel robot designed with the modular concept.

robot therefore consists in the serial arrangement of an active brick with a passive one (see Fig. 2).

Briefly, the kinematical part of the modular concept uses as an input the desired mobility of the robot, i.e. the translational and rotational degrees of freedom which have to be performed ( $T x, T y, T z, R x, R y, R z)$. Then, with the help of a conceptual solution catalogue, this global requirement is transformed into an exhaustive list of all possible combinations of 2-D active and passive bricks fufilling it. At that point, the bricks are expressed only with their degrees of freedom arrangement, independently from any mechanical realisation. The kinematical part of this methodology consequently allows to design a large variety of machines, from machine-tools to microscale robots. The choice of the optimal solution in the exhaustive list is motivated by the specifications of the aimed robot.

The second part of the concept consists in mechanically designing these modular building bricks: for the high precision robots considered in this work, the use of flexure joints is compulsory. Consequently, the aim is to propose a mechanical solution catalogue which, for each brick, lists several possible flexure-based designs. The current research on this modular concept is thus focusing on the generation of this mechanical solution catalogue. The challenges encountered will be presented in the next paragraphs.

\section{Active bricks}

As aforementioned, the active bricks are planar flexure-based mechanisms whose role is to actuate one to three degrees of freedom. The motions which are not actuated are blocked, i.e. the stiffness of the structure along these movements should be at least 100 times higher than the stiffness of the actuated motions (Henein, 2001); practically, a factor of around 1000 is recommended to avoid low transverse eigenfrequencies. The planarity of the mechanisms consists in a noteworthy advantage to achieve this desideratum, as the maximisation of the transverse stiffnesses is much more straightforward than with 3-D structures. The thickness of the planar system can indeed be adjusted to fulfil this requirement without deteriorating the desired strokes. 


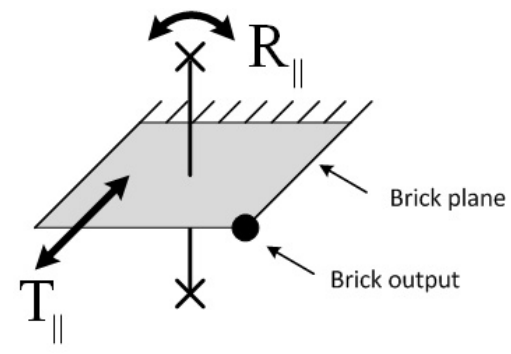

Figure 3. Mobility of the $T_{\|} R_{\perp}$ brick.

As the trend to miniaturise industrial products goes hand in hand with the miniaturisation of the production lines, and thus of the robots (Kobel and Clavel, 2011), the main limitation of the existing industrial high precision machines lies in their bulky volume (of some $\mathrm{dm}^{3}$ ) for the required workspace (usually some $\mathrm{mm}^{3}$ ). Consequently, the chief challenge of the active brick design consists in the maximisation of the ratio between the translational strokes and the characteristic dimension of the robot, which is defined as the highest dimension of the prism enclosing its mechanical parts (see Eq. 1).

$R_{s}=\frac{\text { stroke }[\mathrm{mm}]}{\text { characteristic dimension }[\mathrm{mm}]}$

For instance, the high-precision robots introduced in Sect. 1 present ratios of 0.02 for the Delta ${ }^{3}$ II $( \pm 2 \mathrm{~mm}$ for a characteristic dimension of $200 \mathrm{~mm}$, total volume: $3 \mathrm{dm}^{3}$, see Fig. 1) and of 0.032 for the Sigma $6( \pm 4 \mathrm{~mm}$ for a characteristic dimension of $250 \mathrm{~mm}$, total volume: $6 \mathrm{dm}^{3}$ ). The ideal $R_{S}$ value which this modular concept aims to achieve is around 0.1 , which means performing strokes of $\pm 5 \mathrm{~mm}$ with a cubic robot presenting a characteristic dimension of $100 \mathrm{~mm}$.

\subsection{Remote Centre of Motion (RCM)}

Most high-precision robots and mechanisms performing both rotational and translational motions suffer from the same limitations: besides the low angular strokes, the maximum angle and the maximum translation cannot be achieved at the same time, as in the Sigma 6 robot; its maximal angle of $4^{\circ}$ can indeed be obtained only with a translation of less than $1 \mathrm{~mm}$. This phenomenon is first caused by the strokes of the actuators, which are not sufficient to allow the combined translational and rotational motions. Second, this effect is also induced by the high translational compensatory motions needed during the rotations. This is due to the fact that the centre of rotation does not coincide with the robot output; compensatory translations are thus necessary to keep its position constant during the rotation. A shrewd design making use of a Remote Centre of Motion (RCM) can overcome this problem by virtually locating the rotation centre on the system output, without adding any mechanical part at this point.

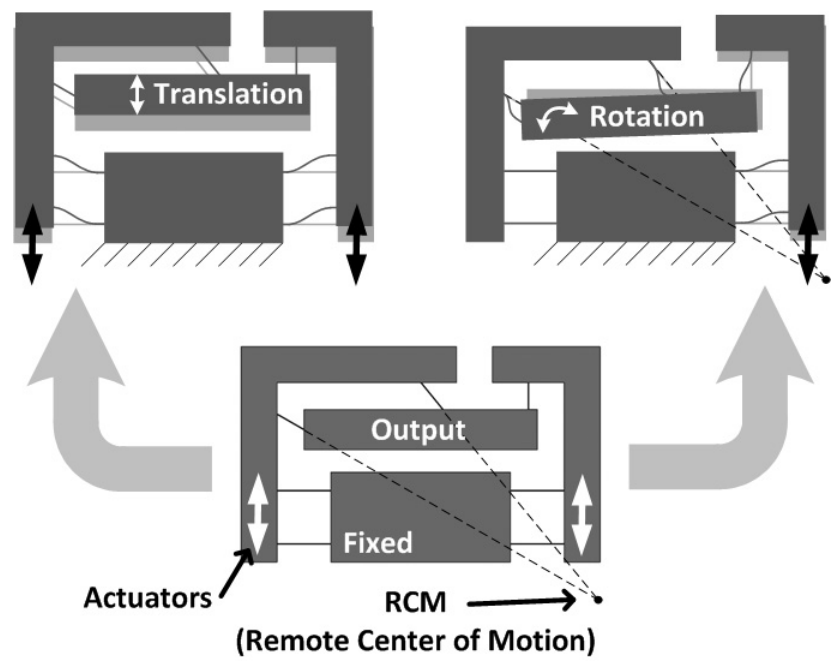

Figure 4. Principle of the designed active $T_{\|} R_{\perp}$ brick: the synchronous motion of the actuators performs the translation, while the rotation around the RCM results from their differential displacement.

To illustrate this solution, the case of the $T_{\|} R_{\perp}$ active brick is now detailed. This planar mechanism performs a translation and a rotation: its notation indicates that the rotation axis is orthogonal to the plane of the brick, whereas the translation is along one direction of that plan (see Fig. 3). According to the definition of the concept, the mechanism comprises two linear actuators: their synchronous motion performs the translation, whereas their differential motion achieves the rotation.

The principle of the mechanical flexure-based design making use of a RCM is shown in Fig. 4, and is illustrated with a machined mock-up with assembled flexure blades (see Fig. 5). The two lower structures are simple 2-blade parallel tables whose function is to guide the actuator moving part (this aspect will be further explained in Sect. 3.2). The position of the left actuator is kept constant during the rotation; both blades which are fixed on the corresponding L-shaped part define the position of the rotation centre, which is located at their virtual intersection. The angle between these two blades should be as near as $90^{\circ}$ as possible in order to maximise the transverse stiffness. The linear motion of the right actuator performs the rotation: through the vertical blade, the force produced by the actuator is transformed into a moment, which causes the output to rotate. In this situation, the lever law $M=F \cdot d$ can be applied, where $M$ is the moment, $F$ is the force, and $d$ is the perpendicular distance between the force and the rotation centre. Consequently, the actuation force which is necessary to rotate the output with a given angle decreases as the distance between the actuator and the RCM increases. Nonetheless, the linear stroke of the actuator which is needed to perform this same angle conversely increases. The distance between the actuator and the 


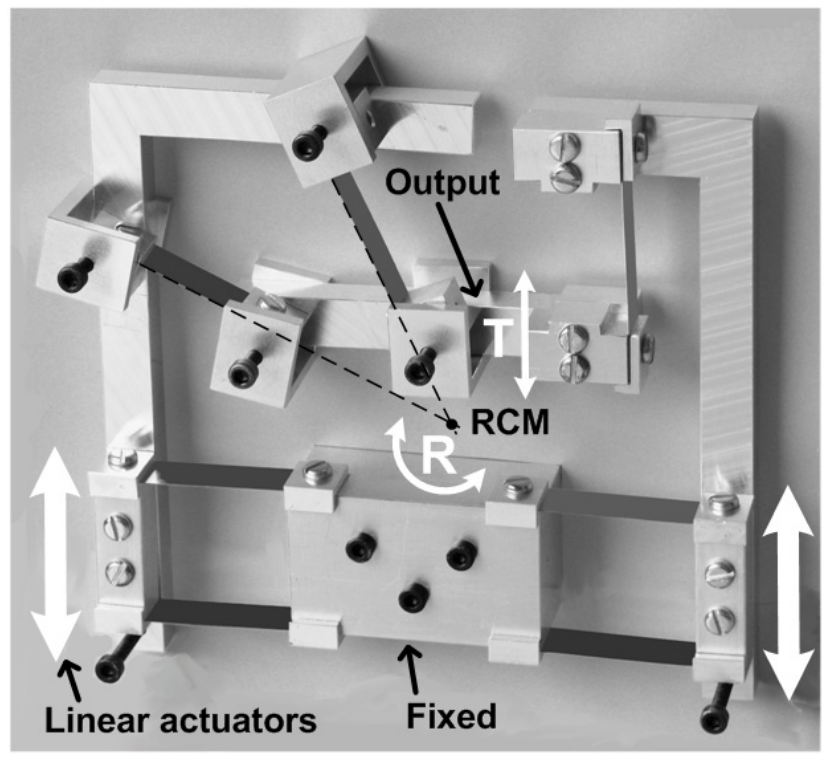

Figure 5. Mock-up of the designed active $T_{\|} R_{\perp}$ brick

RCM should thus be optimised in regard to the robot specifications, as well as to the actuator performances.

This design presents the noteworthy advantage that the location of the rotation centre can be defined at any point of the brick plan. When this brick is integrated into a modular high-precision robot, its RCM must be coincident with the robot output to completely avoid compensatory motions.

\subsection{Actuation sub-brick}

In the frame of this concept of modular flexure-based mechanisms, the actuation of the bricks is always achieved with linear actuators only. Moreover, the guiding of the actuator moving part has to be performed without friction to fulfil the required sub-micrometric precision; a flexure-based structure is thus necessary. Consequently, each active brick comprises the same actuation system, which is composed of the actuator, the guiding structure of the actuator moving part, and a position sensor. This system can be considered as a lower level of modularity, thus defining an actuation sub-brick. The flexure-based mechanisms which transform the linear motion of the actuators into the desired degrees of freedom are then attached to this actuation sub-brick in order to create the desired building brick. The following paragraphs will detail the problematics linked with the guiding system, the choice of the actuators, as well as the mechanical design of the actuation sub-brick.

\subsubsection{Actuator flexure-based guiding mechanism}

A simple flexure-based mechanism performing a translational motion consists in the 4-hinge table (see Fig. 6)

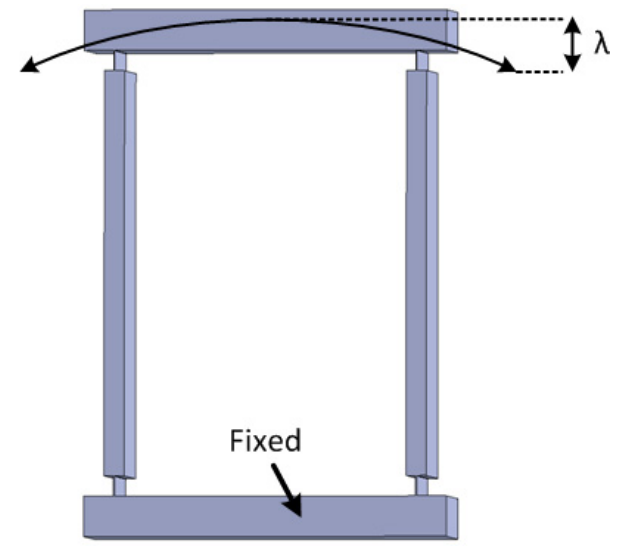

Figure 6. Sketch of the 4-prismatic hinge table trajectory.

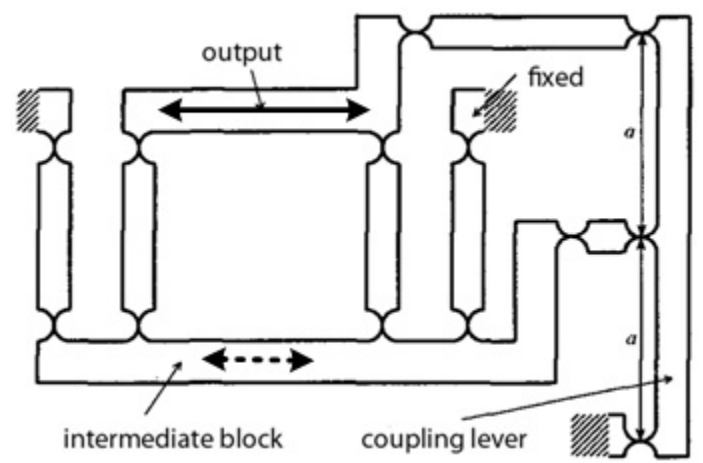

Figure 7. Sketch of the 13-hinge table principle.

(Henein, 2001). Either elliptic, prismatic or truncated circular hinges are conceivable; however, the radius of the circular and elliptic joints necessary to achieve the required stroke is so high that the hinge amounts to a prismatic shape. The critical point of the latter is the stress concentration at the edge of the hinge, which can eventually lead to fracture. To prevent this effect, the edge is rounded: the chosen radius is approximately twice the radius of the wire used for machining.

The crucial issue of this guiding system is its nonrectilinear movement: it indeed performs a translation along a parabolic trajectory (see Fig. 6). For instance, the best designs of a 4-hinge table performing a $\pm 10 \mathrm{~mm}$ stroke within a volume of $10 \times 10 \mathrm{~cm}^{2}$ achieve a parasitic translation, $\lambda$, of $500 \mu \mathrm{m}$ at both ends of the motion. This undesired movement thus becomes problematic for high strokes, as the actuator must withstand the parabolic trajectory of its moving part. The first option to solve this issue is to compensate for the parasitic motion in order to obtain a perfectly rectilinear displacement, making use of a 13-hinge table (see Fig. 7) (Henein, 2001). However, this mechanism is complex and bulky, especially for an actuation sub-brick. Indeed, as the active bricks can actuate up to three degrees of freedom, as many 13-hinge tables should be integrated into the 
same brick, besides the other mechanisms necessary to transform the actuator displacements into the desired motions. Moreover, the force which is necessary to achieve the desired stroke with the 13-hinge table is higher than the force needed with the 4-hinge table, as the number of hinges to deform is increased. This option is thus obviously unconceivable in the frame of this modular concept. The second solution to overcome this issue, which is more straightforward, is to find an actuation principle which is compatible with the parasitic motion of its moving part. The following paragraph will detail the investigation of this second option.

\subsubsection{Actuator problematics}

As the modular robots designed with this concept are intended for industrial use, electromagnetic actuators are selected for their high repetivity relatively to piezo-electric or ultrasonic actuators. Likewise, the moving coil/static magnets configuration is prefered to minimise both the moving mass and the hysteresis created by the moving magnets. Typical values of force requirements for high-precision tasks are continuous forces of $10 \mathrm{~N}$, and peak forces of around $50 \mathrm{~N}$ to allow for high accelerations $(\sim 10 \mathrm{~g})$. Thus, two types of actuators are conceivable: the voice coil and the ironless electronic commutation (EC) actuators.

The voice coil actuator, which is frequently encountered in loudspeakers, consists in a cylindrical arrangement of the moving coil and the static magnets. Like the classical brushed DC (direct current) motors, its control is simple, as the actuation force is directly proportional to the current injected into the coil. However, the value of the parasitic motion of the 4-hinge table is generally higher than the actuator air gap; nonetheless, a simple way to compensate for that limitation is to take advantage of the symmetrical behaviour of the parasitic motion, which is indeed minimal at the equilibrium position of the 4-hinge table (zero stroke) and maximal with the same absolute value at both ends of the stroke. Therefore, the solution consists in eccentrically mounting the coil at zero stroke to make a better use of the available air gap between the coil and the magnets (see Fig. 8).

As for the ironless electronic commutation actuator, it consists in a U-shape frame with fixed magnets and a prismatic moving coil (see Fig. 9). Similarly to brushless DC motors, this 2-phase or 3-phase actuator needs an integrated Hall sensor and a dedicated electronics in order to manage the phase commutation: its control is thus not straightforward. Nonetheless, the main advantage of this solution is its direct compatibility with the parasitic motion of the 4-hinge table. However, the standard EC actuators available on the market are designed for high-power applications, which makes them unsuited for small high-precision mechanisms.

To conclude this actuator problematics, the linear voice coil solution is obviously more adapted to high-precision applications. However, their requirements (strokes, forces) do not match most standard models. The scarce standard actua-

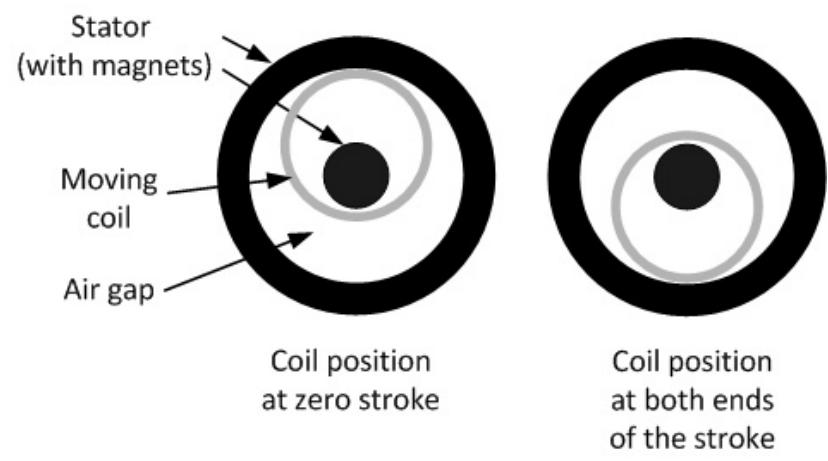

Figure 8. Principle of the eccentric coil mounting (transversal view; the actuation direction is orthogonal to the section).

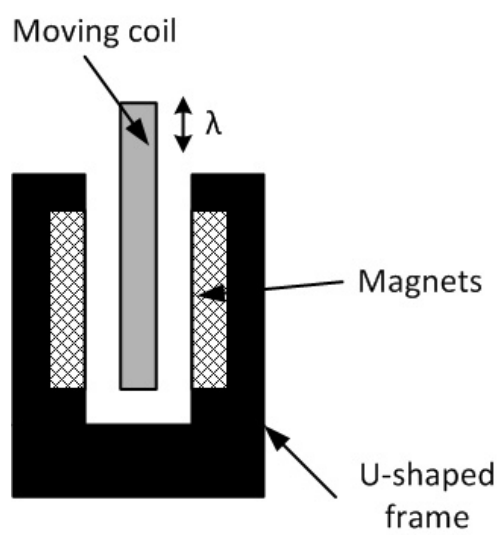

Figure 9. Transversal section of the linear EC actuator (the actuation direction is orthogonal to the section).

tors which fulfil the requirements are bulky and thus difficult to combine with compact high-precision mechanisms, which tend to be as miniaturised as possible. This study of solutions to actuate high-precision systems highlights the lack of fully suitable off the shelf devices; the improvement of the compactness and miniaturisation of flexure-based active mechanisms is thus limited by the unsuitability of the available actuators for the specific requirements of such systems.

\subsubsection{Design of the actuation sub-brick}

A first prototype of the actuation sub-brick has been designed; the selected actuator is a cylindrical voice coil from BEI Kimco Motors (LA15-26-000A, stroke: $\pm 12.7 \mathrm{~mm}$, continuous force: $11.57 \mathrm{~N}$, peak force: $44.48 \mathrm{~N}$ ), which presents an air gap of $390 \mu \mathrm{m}$ on each side of the coil. With an eccentric mounting, as seen in Fig. 8, the parasitic motion of the guiding system must be below $780 \mu \mathrm{m}$. To perform that function, a 4-prismatic hinge table is dimensioned with the following requirements: the planar mechanism should not exceed $10 \times 10 \mathrm{~cm}^{2}$, whereas the desired stroke is $\pm 10 \mathrm{~mm}$. This value is the result of first estimations of the $T_{\|} R_{\perp}$ active 


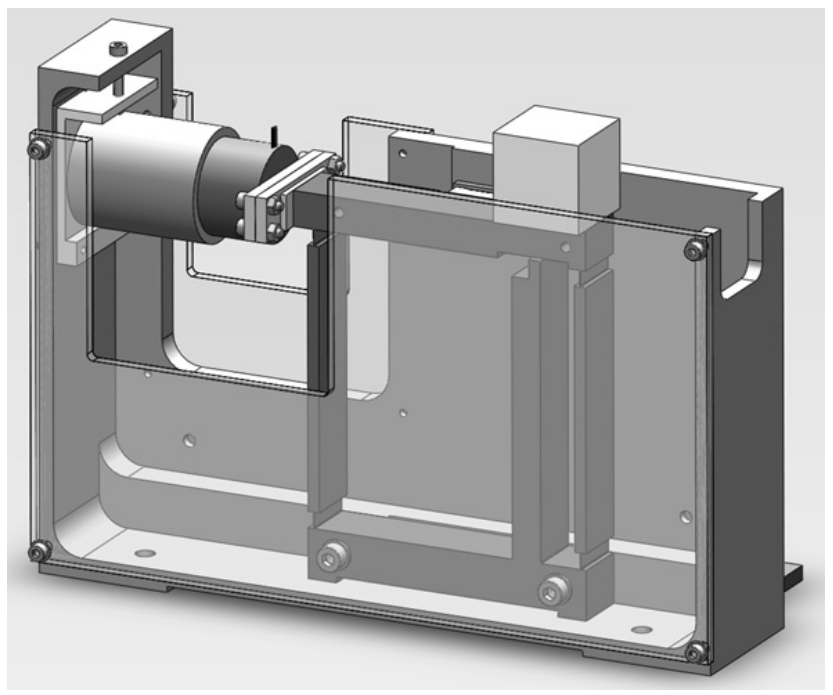

Figure 10. CAD image of the designed actuation sub-brick prototype.

brick described in Sect. 3.1: it allows to perform a translation of $\pm 5 \mathrm{~mm}$ and a rotation of $\pm 10^{\circ}$, the maximum angle being compatible with the maximum linear displacement. Last, the parasitic motion should be minimised. The optimal guiding mechanism design makes use of $250 \mu$ m-thick prismatic hinges, which creates a parasitic motion $\lambda$ reaching $500 \mu \mathrm{m}$ at both ends of the stroke. Finally, an optical encoder from $\mathrm{Nu}$ merik Jena (LIK41, 100× interpolation, resolution: $50 \mathrm{~nm}$ ) is also integrated. Figure 10 shows a CAD view of the final design.

In this design, thermal considerations have also been investigated, on the basis of the work presented in Lubrano and Clavel (2010): first, the conduction of the heat generated by the moving coil into the flexure-based mechanism should be minimised, in order not to deteriorate the repetitivity of the system. To do so, the parts which link the coil to the 4-hinge table have been machined in a material with poor thermal conductivity (namely POM, polyoxymethylene). Then, the guiding system should also be isolated from drafts: plexiglas plates have been designed to perform this function. It is to note that windows have been shrewdly integrated, in order to test a fan cooling around the actuator without influencing the flexure-based mechanism.

As for the feedback device, the aforementioned optical encoder, presenting a resolution of $50 \mathrm{~nm}$, will be used to demonstrate the functionality of the actuation sub-brick. Nonetheless, its characterisation will be performed with more precise measurements: the feedback device will thus be a laser interferometer with a theoretical resolution of $1.25 \mathrm{~nm}$ (SIOS SP-2000 with IS-01 and interpolation modules). Consequently, the limiting resolution of the measurements will be the flatness of the optical surface of the mirror cube used to reflect the interferometer laser beam. The first tests of this prototype will allow to validate this approach and to study the need to integrate optical encoders with a higher resolution, which will obviously be more expensive and more complex to align in the flexure-based system.

As for the planned measurements, besides this validation of the feedback device, the relation between the current injected in the actuator coil and the position of the sub-brick output will be characterised. This will also allow to investigate the possible effects of the coil eccentricity on the voice coil behaviour.

\section{Passive bricks}

Some considerations concerning the design of the passive bricks will now be presented. As seen in Sect. 2, the passive bricks are transmission mechanisms which present from one to five degrees of freedom. These are passive, i.e. free to move but not actuated; the other movements are blocked (see definition in Sect. 3). Like the active bricks, the passive bricks are planar mechanisms; however, axisymmetrical structures, for example cylindrical, are also conceivable. In several cases, such as the mobility illustrated in Fig. 11, an arm-like system is simpler and more efficient than a planar mechanism. The chief challenge of the passive brick design also consists in the maximisation of the strokes: nonetheless, unlike the active case detailed in Sect. 3, this requirement is not necessary for all motions of the brick. Without detailing all kinematical aspects, the passive degrees of freedom can either allow a motion which is required at the robot output or suppress an overconstraint. In the first case, the strokes have to be maximised, because they are directly linked to the robot specifications. In other words, the passive motion must achieve the same displacement as the required stroke on the robot output. However, in the second case, the passive movement only suppresses an overconstraint, i.e. avoids that the position of a blocked degree of freedom is defined by two mechanisms, which would create internal stresses detrimental to the aimed precision. Consequently, the corresponding stroke does not need to be maximised to perform its function. Figures 11 and 12 present two possible designs of passive bricks.

This consideration leads to a reflection on the mechanical solution catalogue, to which the concept of modular flexurebased mechanisms aims. If its validity is undoubted for the active bricks, as the possible designs of these several mechanisms making use of the actuation sub-brick are limited, its usefulness for passive bricks is questionable. First, as these bricks perform from one to five degrees of freedom, the number of possible brick mobilities is higher than for the active bricks. Then, the previous discussion on the role of the passive motions leads to different potential mechanical designs, according to the function of each degree of freedom of the brick. Consequently, the solution catalogue of the passive bricks would present too many solutions to be of any practical use. 


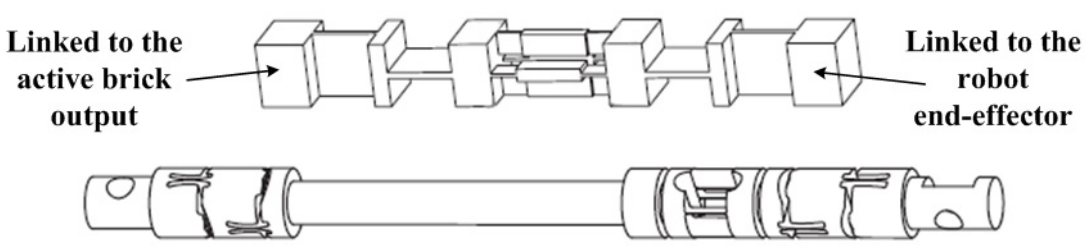

Figure 11. Arms of the Sigma 6 robot (Helmer, 2006), which can be used as passive bricks. They present 5 degrees of freedom; the only blocked motion is the translation along the arm axis.

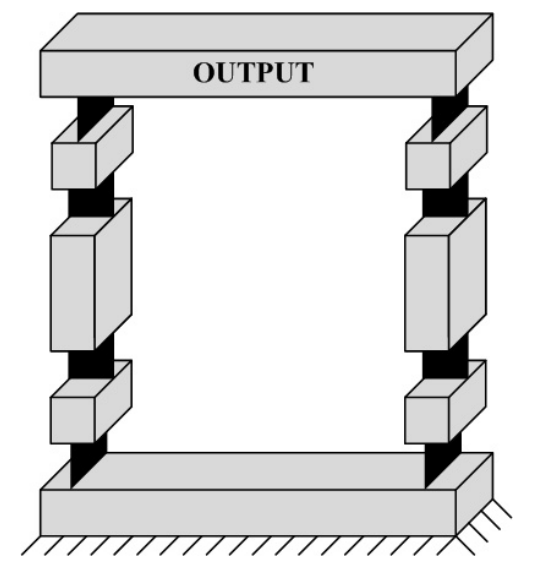

Figure 12. Principle of a possible design of a passive brick performing two rotations and two translations. This mechanism is often referred to as "space parallelogramm".

Finally, the definition of the modular concept states that the kinematic chains of the robot are composed of an active brick and a passive brick which are serially arranged. This point is crucial for the establishment of the conceptual solution catalogue, whose function is to transform a 3-D design problem into several 2-D ones. Nonetheless, when it comes to the mechanical design of the robot, this serial arrangement may not always consist in the optimal solution: both active and passive bricks could be merged in a unique flexure-based structure, which would perform actuated, passive and blocked degrees of freedom. Its design may be simpler and more efficient than the serial arrangement of two separate structures.

To conclude, a judicious mechanical solution catalogue consists in establishing a preferred design for each active brick, and several possibilities for the passive bricks. This catalogue can thus be considered as a useful tool to tackle ultra-high precision flexure-based design, without offering off the shelf solutions. The robot designer will still need to evaluate the validity of the proposed mechanims, and potentially to redesign active, passive, or unique bricks which optimally fulfil the requirements of his/her specific application.

\section{Application of the concept to a 5-DOF robot}

The last part of this paper will illustrate the concept of modular flexure-based mechanisms by detailing a practical example, namely the design of an ultra-high precision 5-DOF robot. The choice of this mobility has been motivated by the micromanipulation and micro-assembly tasks, which consist in typical industrial applications of high-precision robots. These tasks often require five degrees of freedom, namely the three translations $(T x, T y, T z)$ and two rotations $(R x, R y)$. In other words, the only motion which is undesired is the rotation around the axis of the robot tool (which is most of the time vertical, thus $R z$ ). Even though this kinematics is often required for industrial applications, it is complex to design and machine, which explains the scarcity of industrial prototypes.

The specifications of this 5-DOF robot are thus performing three translations and two rotations; the required strokes are $\pm 5 \mathrm{~mm}$ and $\pm 10^{\circ}$, the maximum linear and rotational displacements being achievable at the same time. As for the resolutions, the ideal values are $5 \mathrm{~nm}$ and $0.1 \mu \mathrm{rad}$. The overall volume of the robot should not exceed $1 \mathrm{dm}^{3}$, thus achieving a $R_{s}$ ratio of around 0.1 (see Sect. 3). Eigenfrequencies should also be maximised in order to allow for accelerations up to $10 \mathrm{~g}$.

The first step of the modular concept consists in making use of the conceptual solution catalogue to transform the 3$\mathrm{D}$ design problem into several 2-D brick designs. The kinematical aspects of the methodology being out of the frame of this paper, only the selected robot kinematics is presented (see Fig. 13). The criteria which have been used to choose this structure in the solution catalogue are the following: the number of different building bricks must be minimised, and the passage from this mobility to another one must be simple, i.e. this robot must be easily transformable into a 4-DOF or a 6-DOF one.

Figure 13 shows the 5-DOF kinematics, with the three different 2-D bricks necessary to build this robot. The first active brick, noted $T_{\|}$, consists in a translational motion; its mechanical design is a simple 4-prismatic hinge table. Then, the second active brick is the $T_{\|} R_{\perp}$ mechanism which has been detailed in Sect. 3.1: it performs a translation and a rotation, making use of a Remote Centre of Motion to avoid compensatory motions during the rotation. Last, only one passive 


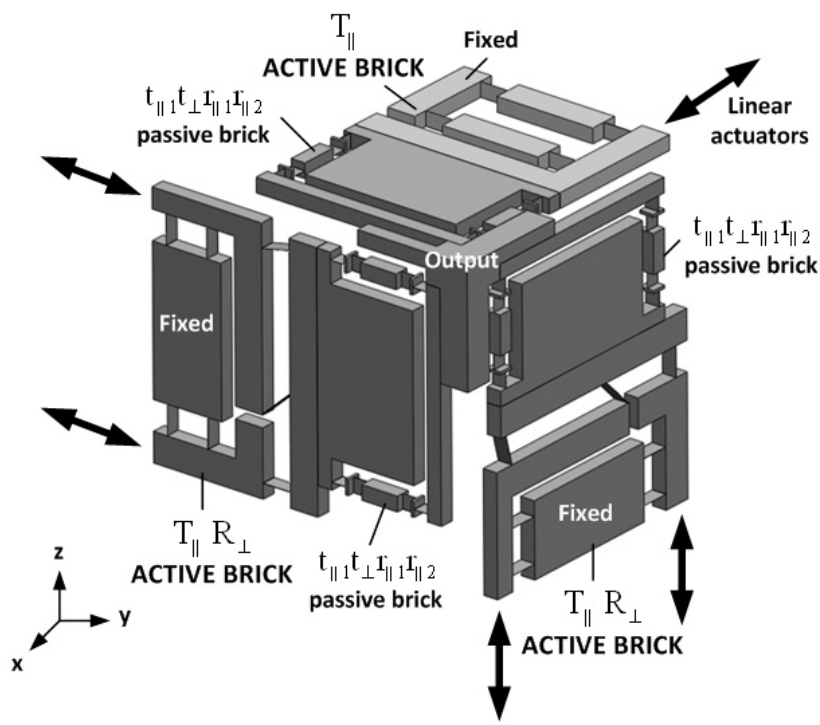

Figure 13. 5-DOF robot kinematics, with sketches of the brick design principles. The notation of the passive bricks is similar to the notation of the active bricks (see 3.1), with lowercase letters to indicate non-actuated motions.

brick $\left(t_{\| 1} t_{\perp} r_{\| 1} r_{\| 2}\right)$ is required, which performs two translations and two rotations. As its notation indicates, one translation is along an axis which is orthogonal to the brick plane, whereas the second is along one axis of the plane. As for the two rotations, their axes correspond to both directions of the brick plane. One possible mechanical design consists in the space parallelogramm illustrated in Fig. 12. A double-scaled mock-up of this 5-DOF robot, named Legolas 5, has been machined with assembled flexure blades in order to validate the design principles (see Fig. 14).

The next step of this robot development is the conception of an industrial prototype; as the bricks will be monolithically machined by W-EDM (Wire-Electro-Discharge Machining), the design must be reviewed in order to be more compact and adapted to this machining process; among other originalities, it includes the aforepresented actuation subbrick, as well as gravity compensation mechanisms.

\section{Summary and conclusions}

A new concept of modular flexure-based mechanisms to design ultra-high precision robots has been presented in this paper. Its crucial advantage over other methodologies is the transformation of a 3-D design problem into several 2-D designs, which are simpler, well-mastered and easy to machine monolithically. A short description of the concept bases has allowed to introduce the representation of the modular parallel robot, as well as both types of planar flexure-based building bricks which are required for its design, namely the active and passive bricks.

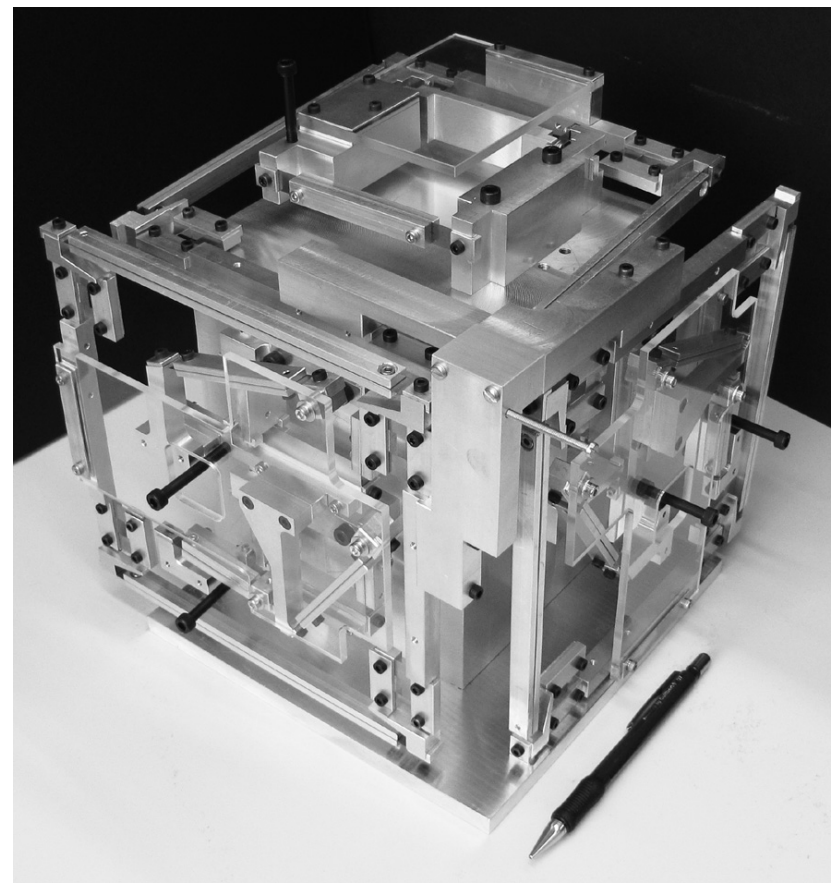

Figure 14. Double-scaled mock-up of the Legolas 5 robot.

The section dedicated to active bricks has detailed the main design challenge, which consists in the maximisation of the strokes. A ratio allowing the comparison between highprecision robots has been introduced, and a design making use of a Remote Centre of Motion has been proposed as a solution to avoid translational compensatory motions during the rotations. Moreover, an actuation sub-brick has been designed: some crucial issues, as the parasitic motion of the actuator moving part guiding system and the actuator choice have been detailed. The problematics of the actuator integration has highlighted the need for actuation solutions specifically intented for high-precision robots and mechanisms. The future work on this sub-brick is its experimental characterisation, which will allow to validate the measurement feedback system, the eccentric mounting of the actuator coil, as well as the thermal isolation of the flexure-based mechanism.

As for the passive bricks, the maximisation of some specific strokes, based on the role of the corresponding degrees of freedom, has been presented. The discussion on the validity of a mechanical solution catalogue has first highlighted that the serial arrangement of the active and passive bricks should be extended, permitting their merging into a unique mechanism. Then, it also allowed to conclude that the solution catalogue should include some possible designs, thus consisting in a useful tool for the designer, but cannot and should not be considered as exhaustive. 
Last, this modular concept has been illustrated with a practical application, consisting in a high-precision 5-DOF robot. A double-scaled mock-up with assembled blades has validated the first design; the development of an industrial prototype, which would among other originalities include the actuation sub-brick, is currently in progress. Its machining and characterisation will allow to prove that the performances of a robot which has been designed with this new methodology are similar or better than the performances of robots resulting from more classical conception procedures.

Acknowledgements. The authors would like to thank the Laboratoire de Systèmes Robotiques collaborators, for their precious advice and the constructive brainstorming sessions, which contributed to the development of this work.

Edited by: A. Barari

Reviewed by: S. Henein and another anonymous referee

\section{References}

Bacher, J.-P., Bottinelli, S., Breguet, J.-M., and Clavel, R.: Delta ${ }^{3}$ : a New Ultra-high Precision Micro-Robot: Design and Control of a Flexure Mechanism, Journal Européen des Systèmes Automatisés, 36, 1263-1275, 2002.

Bottinelli, S., Henein, S., Aymon, C., and Clavel, R.: Movement transmission unit and movement transmission apparatus employing the same, US Patent US006453566B1, 24 September 2002.
Helmer, P.: Conception systématique de structures cinématiques orthogonales pour la micro-robotique, Ph. D. Thesis, No. 3365, Ecole Polytechnique Fédérale de Lausanne (EPFL), 2006.

Helmer, P., Mabillard, Y., Clavel, R., and Bottinelli, S.: High precision apparatus for imposing or measuring a position or a force, US Patent US2004025569A1, 23 December 2004.

Henein, S.: Conception des guidages flexibles, Presses Polytechniques et Universitaires Romandes, Collection Meta, 2001.

Kobel, P. and Clavel, R.: Miniaturization Challenges and Their Impact on the Micro-factory Concept and Manipulators, Japan Society of Precision Engineering, 77, 3, 263-268, 2011.

Lubrano, E. and Clavel, R.: Thermal Calibration of a 3 DOF Ultra High-Precision Robot Operating in Industrial Environment, Proceedings of the 2010 IEEE International Conference on Robotics and Automation, IEEE, 3692-3697, 2010.

Richard, M. and Clavel, R.: A new concept of modular kinematics to design ultra-high precision flexure-based robots, Proceedings for the joint conference of the 41st International Symposium of Robotics and the 6th German Conference on Robotics, Munich, 940-947, 2010.

Tolfree, D.: Commercialising Nanotechnology, concepts - products - markets, International Journal of Nanomanufacturing, 1, 1, 117-133, 2006. 